\title{
Article
}

\section{Eating Behaviors in Healthy Young Adult Twin Pairs Discordant for Body Mass Index}

\author{
Bram J. Berntzen ${ }^{1}$, Sakari Jukarainen ${ }^{1}$, Leonie H. Bogl ${ }^{2,3}$, Aila Rissanen ${ }^{1}$, Jaakko Kaprio ${ }^{3,4}$ and Kirsi H. Pietiläinen ${ }^{1,5}$ \\ ${ }^{1}$ Obesity Research Unit, Research Program for Clinical and Molecular Metabolism, Faculty of Medicine, University of Helsinki, Helsinki, Finland, ${ }^{2}$ Leibniz Institute \\ for Prevention Research and Epidemiology - BIPS, Bremen, Germany, ${ }^{3}$ Institute for Molecular Medicine Finland, FIMM, University of Helsinki, Helsinki, Finland, \\ ${ }^{4}$ Department of Public Health, Finnish Twin Cohort Study, University of Helsinki, Helsinki, Finland and ${ }^{5}$ Endocrinology, Abdominal Center, Obesity Center, \\ Helsinki University Hospital and University of Helsinki, Helsinki, Finland
}

\begin{abstract}
We aimed to study the eating behavioral traits that associate with body mass index (BMI) among BMI-discordant twin pairs. This cross-sectional study examined self-reported eating behaviors in 134 healthy young adult twin pairs (57 monozygotic [MZ] and 77 same-sex dizygotic [DZ]), of whom $29 \mathrm{MZ}$ and $46 \mathrm{DZ}$ pairs were BMI discordant (BMI difference $\geq 3 \mathrm{~kg} / \mathrm{m}^{2}$ ). In both $\mathrm{MZ}$ and $\mathrm{DZ}$ BMI-discordant pairs, the heavier co-twins reported being less capable of regulating their food intake optimally than their leaner co-twins, mainly due to 'frequent overeating'. Furthermore, the heavier co-twins reported augmented 'disinhibited eating', 'binge-eating scores' and 'body dissatisfaction'. The twins agreed more frequently that the heavier co-twins (rather than the leaner co-twins) ate more food in general, and more fatty food, in particular. No significant behavioral differences emerged in BMI-concordant twin pairs. Overeating — measured by 'frequent overeating', 'disinhibited eating' and 'binge-eating score' - was the main behavioral trait associated with higher BMI, independent of genotype and shared environment.
\end{abstract}

Keywords: Twins; body mass index; obesity; eating behavior; disinhibition; overeating

(Received 22 June 2019; accepted 25 June 2019)

Eating behaviors involve dietary and psychological traits in regulation of food intake and weight management. Eating behaviors vary strongly between individuals, and are regulated by complex interactions between physiological, psychological, environmental and genetic factors (Grimm \& Steinle, 2011). Obesity is considered to be primarily caused by overconsumption (Swinburn et al., 2009), which is a plausible consequence of disrupted eating behavioral traits (Bryant et al., 2007; Bublitz et al., 2010; van Strien et al., 2012). Although subjects with obesity seldom self-report higher energy intake than do those at a healthy weight (Goris et al., 2000; Pietiläinen et al., 2010), obesity and increased body mass index (BMI) have, in questionnaires on eating behavioral patterns, been consistently associated with disinhibition of eating (Bryant et al., 2007).

The disinhibited eating measure encompasses social, taste and emotional triggers for overeating (Hyland et al., 1989). Emotional eating (as a result of negative emotions) and external eating (vulnerability to tempting food signals) may moderate the relationship between overeating and weight increase in adults (van Strien et al., 2012). Perhaps as a consequence of weight gain, individuals with obesity are often dissatisfied with their bodies (Weinberger et al., 2016), which in turn may be one motivation to lose weight (Vartanian et al., 2012). A common weight loss approach is dietary restraint; a cognitive effort to self-restrain caloric intake

Author for correspondence: Bram J. Berntzen, Email: bram.berntzen@helsinki.fi Cite this article: Berntzen BJ, Jukarainen S, Bogl LH, Rissanen A, Kaprio J, and Pietiläinen KH. (2019) Eating Behaviors in Healthy Young Adult Twin Pairs Discordant for Body Mass Index. Twin Research and Human Genetics 22: 220-228, https://doi.org/10.1017/thg.2019.43
(Lowe et al., 1991). Its relationship with BMI is complex and ambiguous. Dietary restraint seems to be necessary for the treatment of obesity through energy restriction, though it may increase risk for eating pathology and obesity if practiced inappropriately (Schaumberg et al., 2016).

When investigating predictors of obesity, it is relevant to control for genetic factors. Currently, over 500 genetic loci related to adiposity traits have emerged through genomewide association studies (Loos, 2018), and many of these loci are also associated with eating behaviors (Grimm \& Steinle, 2011).

One can control for genetic factors through the phenotypediscordant monozygotic (MZ) twin pair method (Vitaro et al., 2009), a unique example of a case-control study wherein participants are fully matched for genotype, sex, age and shared environmental factors, but vary in a particular variable such as BMI. Any behavioral differences within MZ twin pairs are plausibly due to environmental experiences and exposures that are unique to one of the twins in that pair. In dizygotic (DZ) twin pairs, behavioral differences result from both environmental and genetic differences because they share approximately $50 \%$ of their segregating genes.

Studies employing an obesity-discordant MZ twin design with twins rating their eating behaviors in relation to their co-twin's (Pietiläinen et al., 2010; Rissanen et al., 2002) have revealed that most twin pairs agree that the co-twins with obesity eat more food overall (Pietiläinen et al., 2010; Rissanen et al., 2002), prefer fatty food (Rissanen et al., 2002) and consume less healthy food (Pietiläinen et al., 2010). This implies that these behaviors are associated with acquired obesity. In another study including both 
$\mathrm{MZ}$ and DZ twins, ingestion of more food in general was the strongest independent correlate of intrapair BMI differences (Bogl et al., 2009).

Overall, most studies have only investigated a limited number of eating behavioral traits in relation to obesity in the same population, and therefore lack a more global view on the patterns behind weight control (French et al., 2012). Several studies have also been unable to control for any genetic influences on the association between eating behaviors and obesity. Building on current knowledge of eating behaviors and obesity by assessing a wide variety of eating behavioral traits within healthy young adult BMI-discordant MZ and DZ twin pairs, we attempted to uncover the eating behavioral traits that are associated with BMI independent of genetic background and of shared environmental factors.

\section{Materials and Methods}

\section{Participants}

This cross-sectional study included 134 young adult twin pairs (57 $\mathrm{MZ}$ and 77 same-sex DZ twin pairs, aged 22-36), of whom $29 \mathrm{MZ}$ and $46 \mathrm{DZ}$ pairs were BMI discordant (BMI difference $\geq 3 \mathrm{~kg} / \mathrm{m}^{2}$ ). The cutoff point for BMI discordance was defined earlier (Hakala et al., 1999; Rönnemaa et al., 1997). The remaining $28 \mathrm{MZ}$ and 31 DZ BMI-concordant twin pairs (BMI difference $<3 \mathrm{~kg} / \mathrm{m}^{2}$ ) functioned as reference groups to compare eating behaviors when BMI within the twin pairs was similar. Recruitment was from 2 population-based longitudinal studies of 10 complete Finnish birth cohorts between 1975-1979 and 1983-1987 (FinnTwin12 and FinnTwin16, $N=5,417$ pairs; Kaprio, 2013), with data retrieved between 2003 and 2013. We took advantage of all the follow-up time points after the age of 20 years from wave 4 in FinnTwin12 (mean age 22 years) and both waves 4 and 5 follow-ups in FinnTwin16 (i.e. ages 25 and 35 years) to find the rare BMI-discordant MZ twins. If the twin pair had attended twice, the latter year was selected. For the DZ twins, we only studied BMIdiscordant pairs from the 25-year follow-up of the FinnTwin 16 because at that age a sufficiently large group was achieved. Additionally, a statistician created an algorithm to randomly select BMI-concordant twin pairs to approximately match the number of discordant twin pairs. Participants were enrolled based on their responses to questions on height and weight at a young adult age, with the aim to cover the full BMI range of subjects with healthy weight and with obesity, and a wide range of intrapair BMI differences. One exclusion criterion for all twins was clinical diagnosis of an eating disorder, or any mental or medical disease, in order to investigate common variations in eating behavioral traits, not those induced by disease or disorder. Informed consent came from all individual participants included in the study. The study was approved by the Ethics Committee of Helsinki University Central Hospital.

\section{Anthropometric Measurement}

Height and weight were measured objectively to calculate BMI. Fat mass and body fat percentage were assessed with dual energy x-ray absorptiometry (DEXA). Zygosity of the twin pairs was confirmed through genotyping of multiple genetic markers from large genotyping arrays with hundreds of thousands of genetic variants (Illumina 670 \& Illumina Human CoreExome chips). More details on anthropometric assessment methods can be found in Jukarainen et al. (2016).

\section{Food Diary}

To create a basic dietary profile, the participants kept a 3 days' food diary ( 2 working days and 1 nonworking day). A registered dietician provided instructions for the dietary intake recording, using the program Diet32 (nowadays AivoDiet) to calculate food consumption and energy intake (Mashie FoodTech Solutions AB, 2017); this is based on 'Fineli'; the Finnish National Food Composition Database (Finnish Food Composition Database, 2009).

\section{Food Intake Regulation}

The twins selected one from four statements about their ability to regulate food intake (Supplementary Text S1), as in earlier studies (Keski-Rahkonen et al., 2005, 2007; Pietiläinen et al., 2010). Shortened descriptions of the answer categories were '1. Optimal eating, 2. Frequent overeating, 3. Frequent restricted eating and 4. Alternating overeating and restriction'. However, due to sparse data for some uncommon behaviors, we collapsed categories 2, 3 and 4 into one category for data analysis, creating a single variable with two values: 'nonoptimal eating' versus 'optimal eating'.

\section{Eating Behavior}

Four eating behavior questionnaires were used in this study. The Three-Factor Eating Questionnaire (TFEQ) investigates the cognitive restraint of eating, disinhibited eating and susceptibility to hunger (Stunkard \& Messick, 1985). These TFEQ outcome measures are further divided into seven subscales: flexible control (gradual and subtle approach of limiting food intake) and rigid control (all-or-nothing approach) (Westenhoefer, 1991); habitual, emotional and situational susceptibility to disinhibition (Bond et al., 2001); and internal locus for hunger (regulated and interpreted internally) and external locus for hunger (triggered by external cues; Bond et al., 2001). The Dutch Eating Behavior Questionnaire (DEBQ) comprises emotional eating, external eating and restrained eating (van Strien et al., 1986). The BingeEating Scale (BES) assesses the severity of and preoccupation with binge-eating (Gormally et al., 1982). Three variables from the Eating Disorder Inventory-2 (EDI-2) included are drive for thinness, body dissatisfaction and bulimia (Garner, 1991).

The DEBQ, TFEQ and EDI-3 (similar to EDI-2) are valid and reliable measures for individuals with overweight and obesity when compared to leaner controls (Bohrer et al., 2015). The BES is a valid and reliable measure for both objective and subjective binge-eating severity (Timmerman, 1999).

\section{Co-Twin Comparison Questionnaire}

Co-twins rated each other's eating behaviors in the previous 12 months through a questionnaire that enquired about 10 dietary intake and related behavioral aspects, answering 'which of you (you or your co-twin) ...'; for example, '... eats more?, ... eats more fatty foods?, ... eats more slowly?' (Supplementary Text S2), see also (Bogl et al., 2009).

\section{Data Analysis}

Stata/SE 13.0 (StataCorp, College Station, TX) served for statistical analyses. Nonparametric statistical tests were performed because of the small sample size and nonnormal distribution of the majority of the data. All statistical tests we performed, unless stated otherwise, within BMI-discordant and -concordant MZ and DZ twin pairs separately. The cutoff point to indicate statistical 
significance was $p<.05$. As not all questionnaire data were complete, a table of the number of twin pairs who completed each questionnaire is in the supplementary material (Supplementary Table S1), which is available on the Cambridge Core website.

Anthropometry and food diary. Intrapair differences in the anthropometric measures were examined with Wilcoxon signed-rank tests, and this test also compared dietary intake and macronutrient proportion in the leaner versus heavier co-twins. Anthropometry measures were compared between leaner MZ and DZ co-twins, and heavier MZ and DZ co-twins with Mann-Whitney $U$ tests. Calorie intake and relative consumption of macronutrients (fat, protein, carbohydrates and alcohol) in grams per day, and in percentages of energy intake, were calculated according to Fineli (Finnish Food Composition Database, 2009). All other dietary components appeared as grams consumed per day.

Food intake regulation. The prevalence of optimal eating and nonoptimal eating between leaner and heavier co-twins was examined by McNemar's test. Prevalence of optimal and nonoptimal eating was reported, as well as absolute prevalence differences.

Eating behaviors. Scores on the separate domains of the TFEQ, DEBQ, BES and EDI-2 were adjusted to a scale of 0-100 for easier interpretation and comparison (Lauzon et al., 2004), which means that the lowest possible score was subtracted from the actual score and divided by the possible score range, multiplied by 100 (Lauzon et al., 2004). For example, suppose the total score ranges from 12 to 40. If someone scored 26 , then the calculation would be (26 (actual score) -12 (lowest score possible) $) \div(40-12$ (score range) $) \times$ $100=50$. The original cutoff points for interpretation of the BES score were 'severe binge-eating if BES score $\geq 27$, moderate bingeing, 18-26, and no bingeing, $\leq 17$ ' [24]. The new scale of $0-100$ gave as cutoff points 'severe binge-eating if BES score $\geq 59$, moderate bingeing, $38-58$, and no bingeing, $\leq 37$ '. The other questionnaires were evaluated as higher scores reflecting more extreme behavior.

First, survey regression analyses assessed coefficients for the association between standardized behavioral traits (i.e. divided by standard deviation) and BMI as a continuous variable in all twin individuals. A correction was applied for the familial grouping of traits, with age and sex included as covariates. BMI, because of its intuitive interpretation, was not standardized. Behavior standardization enabled equal comparison between associations with BMI.

Subsequently, we analyzed the differences in responses on the TFEQ, DEBQ, BES and EDI-2 questionnaires between leaner and heavier co-twins with Wilcoxon signed-rank tests. We quantified the size of the significant differences with the common language effect size (McGraw \& Wong, 1992). This effect size identifies those cases in which the heavier co-twin scores higher on a behavioral trait than does the leaner co-twin as a proportion of the total twin pairs. Thus, put simply: an effect size of 0.68 for emotional eating signifies that the chance is $68 \%$ that in any random twin pair, the heavier co-twin experiences a higher level of emotional eating. Importantly, an effect size of 0.50 implies that any difference between co-twins is due solely to chance. Hence, an effect size above 0.50 implies a probability superior to chance that the heavier co-twin performs a behavioral trait more strongly, whereas below 0.50 , the heavier co-twin is less likely to do so. We calculated approximate confidence intervals (CI) for effect sizes, as discussed in more detail elsewhere (Altman \& Bland, 2011).
Additionally, we created a correlation matrix of all eating behavioral traits — with a correction for familial clustering — to obtain a better understanding of the overlap or similarity between traits.

Co-Twin comparison questionnaire. We analyzed the co-twin comparison questionnaire separately for $\mathrm{MZ}$ and $\mathrm{DZ}$ twins, but we combined BMI-discordant and -concordant twins in two ways, with Wilcoxon signed-rank tests and multivariate regression analyses, as earlier (Bogl et al., 2009).

Only those twin pairs who provided internally consistent answers as to who performed a particular eating behavior more strongly were included in the Wilcoxon signed-rank tests. The twin pairs were separated into Twin1 (who performed the behavior more strongly according to both co-twins of the pair), and Twin2 (who performed the behavior to a lesser extent). Wilcoxon signed-rank tests compared the differences between the average BMI of Twin1 and Twin2 for all eating behavioral traits, providing the mean difference in BMI $\left(\mathrm{kg} / \mathrm{m}^{2}\right)$ for each eating behavioral trait.

Multivariate regression analyses were performed in all twin pairs. A twin pair was coded -1 if both co-twins agreed that the leaner co-twin performed the behavior, +1 if both agreed the heavier co-twin performed the behavior and 0 in all other cases. This allowed linkage of independent eating behavior to intrapair differences in BMI (BMI heavier co-twin-BMI leaner co-twin), while controlling for age and sex.

\section{Results}

\section{Characteristics and Dietary Profile in Leaner Versus Heavier Co-Twins}

All adiposity measures were higher in the heavier co-twins of $\mathrm{MZ}$ and DZ pairs discordant for BMI (Table 1), as expected with this study design. The leaner co-twins of the MZ twins were on average in the overweight category, and the heavier co-twins in the obesity class 1 category. In the DZ twin pairs, the leaner co-twins on average were of a healthy weight and the heavier co-twins were overweight. Moreover, in the BMI-concordant twins, small intrapair differences in adiposity were evident because of the division into leaner and heavier co-twins (Supplementary Table S2). An overview of all BMI category (e.g. overweight, obesity class 1) comparisons in the whole cohort, and separately by zygosity and BMI discordance is available (Supplementary Table S3).

In BMI-discordant twin pairs, both leaner and heavier MZ co-twins had a higher age, BMI, fat mass and fat percentage than the leaner and heavier DZ co-twins (Supplementary Table S4), and higher weight in leaner MZ co-twins only. Sex and height followed similar patterns between MZ and DZ co-twins. No evidence was present for any difference in BMI-concordant twin pairs between leaner MZ and DZ co-twins or heavier MZ and DZ co-twins.

The food diaries did not reveal any meaningful differences in caloric intake or relative intake of macronutrients between leaner and heavier co-twins in any of the groups (Supplementary Table S5).

\section{Food Intake Regulation in Leaner Versus Heavier Co-Twins}

In MZ and DZ BMI-discordant twin pairs, McNemar's test indicated that regarding food intake regulation, leaner and heavier co-twins differed (MZ: $\chi^{2}=7.36, \quad p=.01 ; \quad \mathrm{DZ}: \chi^{2}=9.31$, $p=.003$; Figure 1$)$. The nonoptimal eating prevalence in leaner versus heavier co-twins was $52 \%$ versus $83 \%$ in $\mathrm{MZ}$ pairs, and 
Table 1. Intra-pair differences in characteristics of MZ and DZ twin pairs discordant for BMI

\begin{tabular}{|c|c|c|c|c|c|c|c|c|}
\hline & \multicolumn{6}{|c|}{ BMI-discordant twin pairs } & \multirow[b]{3}{*}{$\Delta \%$} & \multirow[b]{3}{*}{$p$ value } \\
\hline & \multicolumn{2}{|c|}{$\mathrm{MZ}(n=29)$} & \multirow[b]{2}{*}{$\Delta \%$} & \multirow[b]{2}{*}{$p$ value } & \multicolumn{2}{|c|}{$\mathrm{DZ}(n=46)$} & & \\
\hline & Leaner & Heavier & & & Leaner & Heavier & & \\
\hline Age, y & $30.1 \pm 0.9$ & $30.0 \pm 0.9$ & - & - & $27.4 \pm 0.3$ & $27.5 \pm 0.3$ & - & - \\
\hline Female/male, freq. & $19 / 10$ & $19 / 10$ & - & - & $21 / 25$ & $21 / 25$ & - & - \\
\hline Height, $\mathrm{cm}$ & $172.6 \pm 2.1$ & $172.9 \pm 2.0$ & 0.2 & .52 & $173.3 \pm 1.2$ & $174.8 \pm 1.3$ & 0.9 & .12 \\
\hline Weight, kg & $76.6 \pm 3.4$ & $94.9 \pm 3.9$ & 23.9 & $<.001$ & $65.0 \pm 1.4$ & $87.6 \pm 1.9$ & 35.0 & $<.001$ \\
\hline BMI, $\mathrm{kg} / \mathrm{m}^{2}$ & $25.6 \pm 1.0$ & $31.6 \pm 1.1$ & 23.4 & $<.001$ & $21.5 \pm 0.4$ & $28.7 \pm 0.6$ & 33.5 & $<.001$ \\
\hline Fat mass, kg & $25.6 \pm 2.2$ & $39.3 \pm 2.2$ & 53.5 & $<.001$ & $14.8 \pm 1.2$ & $31.1 \pm 1.7$ & 110.1 & $<.001$ \\
\hline Body fat, $\%$ & $32.3 \pm 1.9$ & $41.4 \pm 1.4$ & 28.2 & $<.001$ & $22.3 \pm 1.6$ & $35.2 \pm 1.6$ & 57.8 & $<.001$ \\
\hline
\end{tabular}

Note: Values are mean \pm standard error.

$\mathrm{BMI}=$ body mass index, $\mathrm{MZ}=$ monozygotic, $\mathrm{n}=$ number of pairs, $\mathrm{DZ}=$ dizygotic, $\Delta \%=$ difference in percentages $[($ heavier - leaner $) /$ leaner $\times 100]$, freq. $=$ frequency.

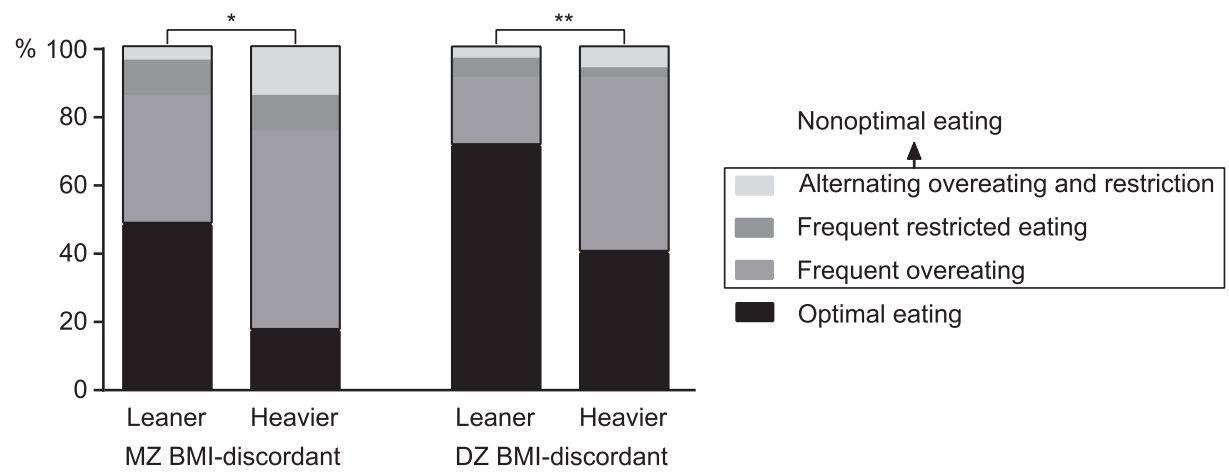

Fig. 1. Percentages of food intake regulation categories in leaner and heavier monozygotic (MZ) and dizygotic (DZ) twins discordant for body mass index (BMI). Note: McNemar's test ${ }^{\star} p<.05,{ }^{\star \star} p<.01$.
$29 \%$ versus $60 \%$ in DZ pairs. Thus, in both MZ and DZ pairs, the absolute prevalence of nonoptimal eating was $31 \%$ higher in the heavier co-twins. Less than half of the leaner MZ (48\%), but the majority of leaner DZ (71\%) co-twins ate optimally. The majority of the heavier co-twins in the MZ (59\%) and DZ (51\%) BMI-discordant groups frequently overate. Only a few individuals in all groups frequently restricted their food intake (3-13\%). In the BMI-concordant groups, leaner and heavier co-twins (58-71\%) mainly ate optimally (Supplementary Figure S1), and thus did not differ in food intake regulation.

\section{Eating Behaviors in Leaner Versus Heavier Co-Twins}

The $p$ values from survey regression analyses in all twin individuals demonstrated strong evidence for the presence of associations of standardized disinhibited eating, restrained eating, binge-eating score, drive for thinness, body dissatisfaction and bulimia with BMI as a continuous variable (Table 2).

In BMI-discordant MZ and DZ twin pairs, evidence was present for higher disinhibited eating (TFEQ), binge-eating scores (BES; $p=.050$ in $\mathrm{MZ}$ pairs) and body dissatisfaction (EDI-2) in the heavier co-twins (Figure 2). Only in DZ twins did the heavier co-twins show higher restrained eating (DEBQ), and drive for thinness (EDI-2). No important intrapair differences appeared in the BMI-concordant groups (Supplementary Figure S2).

The common language effect size for disinhibited eating in $\mathrm{MZ}$ BMI-discordant twin pairs was 0.74 (effect size 95\% CI [0.57, 0.95]) and in DZ twin pairs 0.76 (95\% CI [0.62, 0.94]). The effect size for binge-eating score in $\mathrm{MZ}$ twin pairs was 0.71 (95\% CI [0.50, 1.001]) and in DZ twin pairs 0.73 (95\% CI [0.58, 0.92]), and for body dissatisfaction in $\mathrm{MZ}$ twin pairs this was 0.73 (95\% CI [0.54, 0.99]) and in DZ pairs 0.81 (95\% CI [0.72, 0.91]).

In DZ BMI-discordant female twins, the intrapair differences in body dissatisfaction and bulimia were significantly larger than in male twins, which were the only sex differences among all groups (Supplementary Table S6).

The behavioral traits had mostly negligible and low intercorrelations (although $p$ values showed evidence for associations between traits), aside from three moderate correlation coefficients (Supplementary Table S7).

After further division of the TFEQ outcome measures into seven subscales (Figure 3), the leaner co-twins of the MZ BMI-discordant twin pairs showed significantly higher flexible control. The effect size for flexible control was $0.28(95 \%$ CI $[0.08,0.95])$. The heavier co-twins of this group demonstrated particularly stronger habitual disinhibition (Figure 3), for which the effect size was 0.78 (95\% CI $[0.65,0.93])$. No significant differences were present in the DZ BMI-discordant twin pairs. In BMI-concordant $\mathrm{MZ}$ twin pairs, a stronger flexible control of the leaner co-twins was found (Supplementary Figure S3), with an effect size of 0.21 (95\% CI [0.04, 0.99]).

\section{Leaner and Heavier Co-Twins' Judgment of Each Other's Eating Behaviors}

In the co-twin comparison questionnaire, the twins rated their own eating behaviors in comparison to their co-twin's eating behaviors (Figure 4); for example, 'Which of you (you or your co-twin) eats more?' (Supplementary Text S2). In panel A of Figure 4, the BMI of only those twin pairs who gave the same, internally consistent 
Table 2. Survey regression coefficients of the association between standardized eating behavioral traits and $\mathrm{BMI}$ as a continuous variable

\begin{tabular}{llcl}
\hline \multicolumn{4}{c}{ BMI of individual twins } \\
\hline TFEQ & $\beta[95 \% \mathrm{Cl}]$ & $p$ value & $n$ \\
\hline Cognitive restraint & $0.1[-0.7,0.8]$ & .85 & 176 \\
\hline Disinhibited eating & $1.7[1.0,2.5]$ & $<.001$ & 176 \\
\hline Hunger susceptibility & $0.1[-0.7,0.9]$ & .78 & 176 \\
\hline DEBQ & & & \\
\hline Restrained eating & $1.3[0.6,2.0]$ & $<.001$ & 245 \\
\hline External eating & $0.2[-0.4,0.9]$ & .50 & 248 \\
\hline Emotional eating & $0.6[-0.04,1.3]$ & .07 & 247 \\
\hline BES & & & \\
\hline Binge-eating score & $1.8[1.2,2.5]$ & $<.001$ & 268 \\
\hline EDI-2 & & & \\
\hline Drive for thinness & $1.5[-0.7,2.3]$ & $<.001$ & 255 \\
\hline Body dissatisfaction & $3.2[2.5,3.9]$ & $<.001$ & 255 \\
\hline Bulimia & $0.9[0.2,1.5]$ & .01 & 258 \\
\hline
\end{tabular}

Note: $\mathrm{n}=$ number of individuals, BMI =body mass index, TFEQ $=$ Three-Factor Eating Questionnaire, DEBQ $=$ Dutch Eating Behavior Questionnaire, BES $=$ Binge-Eating Scale, EDI-2 = Eating Disorder Inventory-2, $\beta[95 \% \mathrm{Cl}]=$ regression coefficient with $95 \%$ confidence interval from survey regressions. response on which co-twin performs the behavior more strongly were compared with the Wilcoxon signed-rank test. The number of twin pairs who agreed on which of them performed a behavioral trait more strongly varied per trait (ranging from 10 to 26 out of $55 \mathrm{MZ}$ and from 13 to 27 out of $65 \mathrm{DZ}$ twin pairs). The strongest significant effects on BMI were for the MZ twins who ate more food $\left(+5.2 \mathrm{~kg} / \mathrm{m}^{2}\right)$, more fatty food $\left(+4.4 \mathrm{~kg} / \mathrm{m}^{2}\right)$, snacks $\left(+4.0 \mathrm{~kg} / \mathrm{m}^{2}\right)$, and healthy food $\left(-4.7 \mathrm{~kg} / \mathrm{m}^{2}\right)$, and were more worried about their appearance $\left(-5.2 \mathrm{~kg} / \mathrm{m}^{2}\right)$, as well as smaller but significant findings for eating more sweet and fatty delicacies $\left(+2.3 \mathrm{~kg} / \mathrm{m}^{2}\right)$, eating more regularly $\left(-2.6 \mathrm{~kg} / \mathrm{m}^{2}\right)$ and more slowly $\left(-2.3 \mathrm{~kg} / \mathrm{m}^{2}\right)$. In the DZ twins, significant associations with BMI were for eating more food $\left(+4.9 \mathrm{~kg} / \mathrm{m}^{2}\right)$, fatty food $\left(+3.5 \mathrm{~kg} / \mathrm{m}^{2}\right)$ and snacks $\left(+3.6 \mathrm{~kg} / \mathrm{m}^{2}\right)$.

In panel B of Figure 4, all twin pairs were included for multivariate regression analyses adjusted for age and sex. Intrapair comparisons of several eating behavioral traits were associated with BMI differences. Eating more food and more fatty food were linked to an intrapair difference in BMI of +2.3 and $+2.4 \mathrm{~kg} / \mathrm{m}^{2}$ in $\mathrm{MZ}$ twins, and +2.3 and $+2.6 \mathrm{~kg} / \mathrm{m}^{2}$ in DZ twins. Furthermore, in $\mathrm{MZ}$ twins, eating more snacks was linked to a BMI difference of $+1.8 \mathrm{~kg} / \mathrm{m}^{2}$, whereas eating more healthy food and eating more regularly, as well as being more worried about one's appearance were associated with negative BMI differences $(-2.4,-1.8$ and $-2.7 \mathrm{~kg} / \mathrm{m}^{2}$ ).

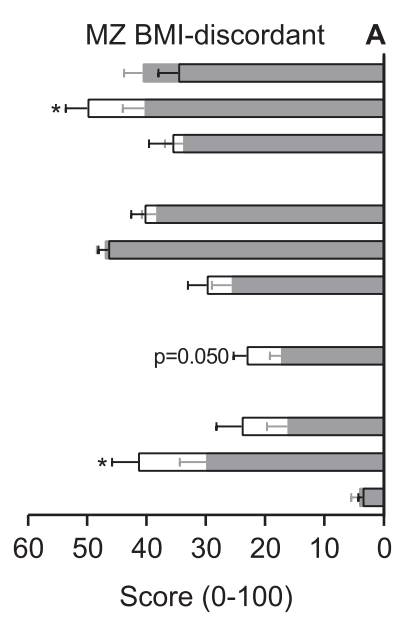
A
TFEQ reating Disinhibited eating Susceptibility to hunger $D E B Q$
Restrained eating
External eating
Emotional eating BES
Binge-eating score EDI-2
Drive for thinness
Body dissatisfaction Bulimia

\begin{tabular}{|l|}
$\square$ \\
$\square$ Leaner co-twins \\
$\square$ Heavier co-twins
\end{tabular}

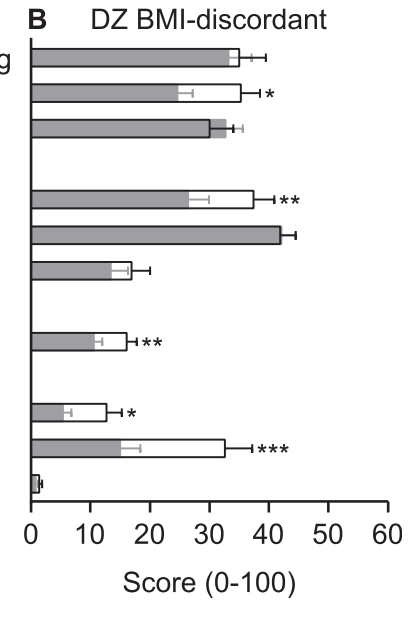

Fig. 2. Overlay bar graph with mean \pm standard error scores on eating behavioral traits in leaner and heavier (Panel A) monozygotic (MZ) and (Panel B) dizygotic (DZ) co-twins in pairs discordant for body mass index (BMI). Note: TFEQ = Three Factor Eating Questionnaire, DEBQ = Dutch Eating Behavior Questionnaire, BES = Binge-Eating Scale, EDI-2 = Eating Disorder Inventory-2. Wilcoxon signed-rank test ${ }^{\star} p<.05,{ }^{\star \star} p<.01,{ }^{\star \star \star} p<.001$.

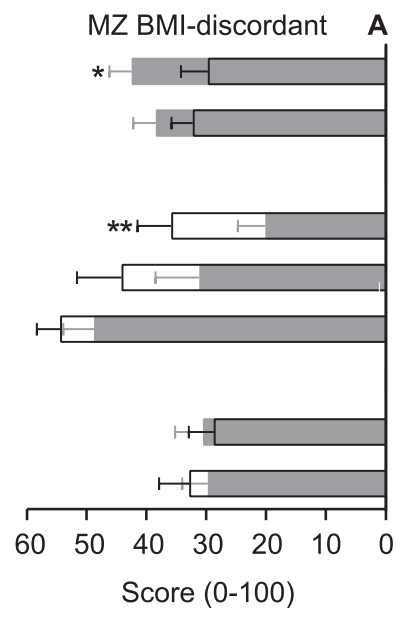

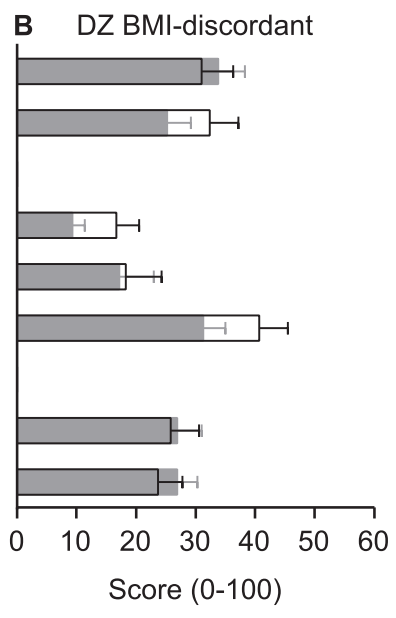

Fig. 3. Overlay bar graph with mean \pm standard error scores on subscales of the Three-Factor Eating Questionnaire in leaner and heavier (panel A) monozygotic (MZ) and (Panel B) dizygotic (DZ) co-twins in pairs who are discordant for body mass index (BMI). Note: Wilcoxon signed-rank test ${ }^{\star} p<.05,{ }^{\star *} p<.01$. 
Which of you, you or your co-twin:
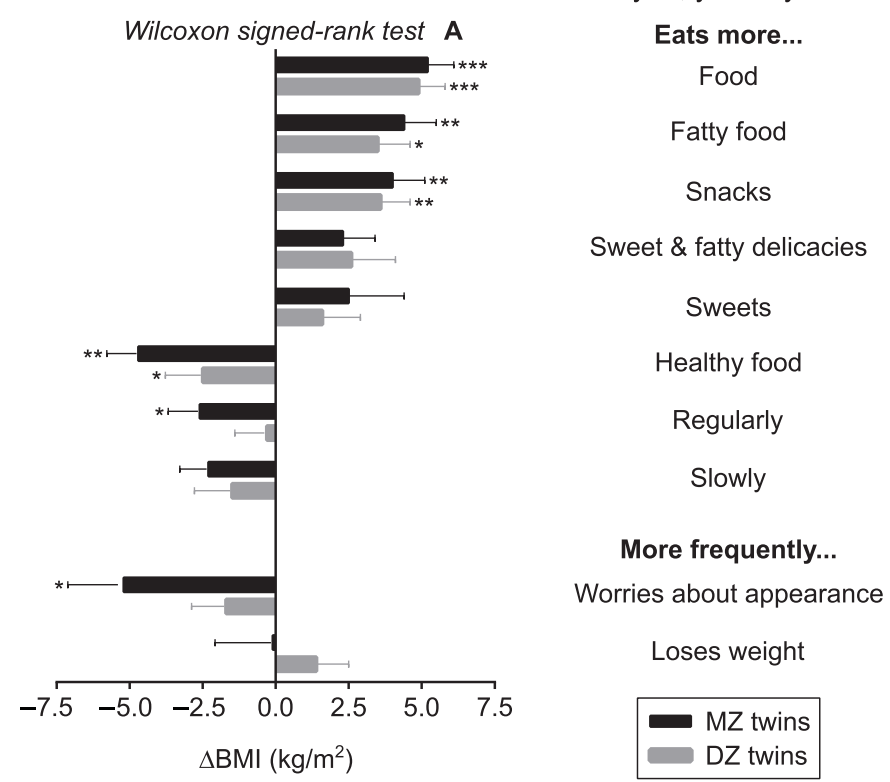

\section{Discussion}

In both MZ and DZ BMI-discordant twin pairs, the heavier co-twins reported difficulties regulating their food intake optimally, and they also reported overall unhealthier eating behavioral traits than did their leaner counterparts. Both twins in such pairs more frequently agreed that the heavier co-twins ate more food and fatty food than did their leaner co-twins, and that in MZ twins the heavier co-twins exhibited an overall unhealthier eating pattern. In BMI-concordant twin pairs, the leaner and heavier co-twins had comparable eating behavior. The discussion will focus on BMI-discordant twin pairs, unless stated otherwise.

Initially, we enquired whether the twins were capable of consuming an appropriate amount of food within the twins' perceived requirements. The majority of the heavier $\mathrm{MZ}$ and $\mathrm{DZ}$ co-twins reported being less capable of eating according to their needs. Instead, they characterized their primary behavior as frequent overeating, in line with our previous findings (Pietiläinen et al., 2010). Notably, in the current study, more than half of the leaner $\mathrm{MZ}$ co-twins self-reported nonoptimal eating. The reason may be that even the leaner $\mathrm{MZ}$ co-twins experienced being overweight on average, and perhaps therefore displayed unhealthier behavioral traits. Another preceding investigation of this question demonstrated that both restrictive and overeating behaviors increased the risk for obesity (Keski-Rahkonen et al., 2007). Overall, studies in naturalistic settings confirm the common co-occurrence of overeating and restraint, but primarily support the beneficial effects of restraint in reducing overeating and promoting weight loss (Johnson et al., 2012; Schaumberg et al., 2016). The current findings also support the association of overeating, rather than food restriction, with a higher BMI, independent of genotype and shared environment.

Augmented disinhibited eating (TFEQ) and binge-eating scores (BES) in the heavier MZ and DZ co-twins further revealed the association between overeating and increased BMI. Disinhibited eating has been linked to BMI (Bryant et al., 2007), and the current study adds evidence for this association independent of genetic and shared environmental factors. Important to note is that the mean

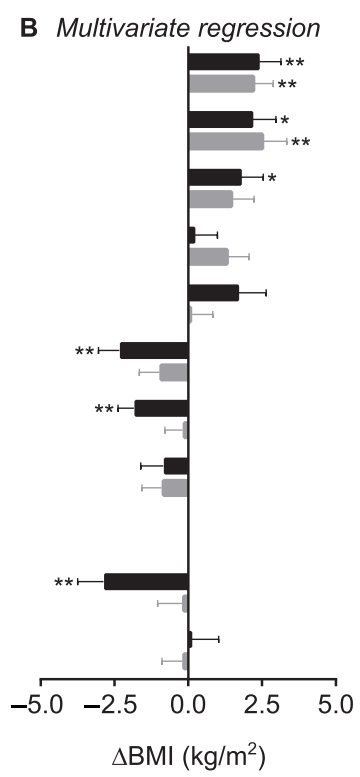

Fig. 4. (Panel A) Wilcoxon signed-rank test was used to compare body mass index (BMI) within monozygotic (MZ) and dizygotic (DZ) twin pairs who gave an internally consistent answer; (Panel B) Multivariate regression analyses were performed within all twin pairs, and indicated the association ( $\beta \pm$ standard error) between co-twin differences in eating behaviors and intrapair differences in BMI ( $\triangle \mathrm{BMI})$ in $\mathrm{kg} / \mathrm{m}^{2}$, controlled for age and sex. Note: ${ }^{\star} p<.05$, ${ }^{\star \star} p<.01,{ }^{\star \star \star} p<.001$.

value of the binge-eating score implies that the participants are nonbingers. This was in accordance with our exclusion of those with eating disorders. A nonbinger might still overeat, but without a dysphoric response (Gormally et al., 1982).

Disinhibited eating was divided into habitual, situational and emotional disinhibition subscales (Bond et al., 2001). These provide more detailed information on the nature of disinhibited eating, which may facilitate the tailoring of interventions. Of all seven TFEQ subscales, habitual disinhibition has most strongly predicted weight gain over 20 years (Hays \& Roberts, 2008). For us, the heavier co-twins of the BMI-discordant MZ but not DZ twin pairs showed higher habitual disinhibition. As this finding was not consistent for both zygosities, no inferences on genetic influence are possible.

We also investigated two restrictive eating behaviors: restrained eating (DEBQ) and cognitive restraint of eating (TFEQ). Both mainly target restrictions from desired, rather than required, ingestion of food (Lowe \& Levine, 2005; van Strien, 2008). Hence, high scores on these restraint measures are no guarantee that individuals are restricting their food intake appropriately to lose weight. Furthermore, restrained eating (DEBQ) measures an intention to restrict food intake, whereas cognitive restraint of eating (TFEQ) measures actual caloric restraint (Williamson et al., 2007). We found restrained eating (DEBQ) to characterize the heavier rather than the leaner co-twins of the DZ twin pairs. However, the cognitive restraint of eating (TFEQ) did not differ within the pairs with either of the zygosities. This suggests that the heavier DZ co-twins here had the intention to restrict, but did not actually restrict food intake. Therefore, they might have intended to incorporate restrained eating as a compensatory mechanism for overeating. We cannot, however, exclude the possibility that restrained eating initiated disinhibited eating for those individuals with high scores on both scales (Ouwens et al., 2003).

We divided cognitive restraint of eating (TFEQ) into two subscales; flexible control and rigid control of eating behavior. Flexible control is a more gradual and subtle approach to limiting food intake than is the all-or-nothing approach of rigid control 
(Westenhoefer, 1991). Rigid control methods include strict consumption rules, which, when broken, may initiate a loss of control of eating (disinhibited eating). Flexible control is known to be linked with decreased eating behavior disturbances, decreased body weight and increased success in weight loss and maintenance, as opposed to the negative health consequences of rigid control (Westenhoefer et al., 1999). Our findings support the view that flexible control may contribute to the BMI difference, at least within the MZ twin pairs. Flexible control was augmented in the leaner co-twins of the BMI-discordant and -concordant MZ twin pairs, even though the overarching cognitive restraint did not differ within the pairs.

The heavier co-twins reported higher body dissatisfaction (in both $\mathrm{MZ}$ and $\mathrm{DZ}$ pairs), and a stronger drive for thinness (in DZ pairs). Both traits have previously been associated with larger body size (Keski-Rahkonen et al., 2005), and we can complement this with our finding that body dissatisfaction was associated with BMI independent of genotype and shared environment. The intrapair differences on the EDI-2 questionnaire were significantly larger for DZ females than for males. This was expected, because body dissatisfaction in those who have obesity compared to normal-weight individuals has been recognized to be considerably higher in women than in men (Weinberger et al., 2016).

The co-twin comparison questionnaire included both BMIdiscordant and -concordant twin pairs, and asked all twins to compare their own behavior with their co-twin's behavior, as in previous studies (Bogl et al., 2009; Pietiläinen et al., 2010; Rissanen et al., 2002). This approach is advantageous because it provides a verification of behavioral traits by the co-twins, who are reliable proxies of each other's behaviors (Hamilton \& Mack, 2000). In our study, the percentage of agreement, within pairs, on which co-twin performs which behavior more strongly is relatively low, this may be because only 2 out of 16 possible answer combinations defined an agreement in the direction of either co-twin. Within the disagreement proportion, the answers were diluted over the remaining 14 answer combinations. Regardless, both MZ and DZ twin pairs agreed more frequently that the heavier co-twin ate more food in general, and more fatty food in particular than their leaner counterparts, in comparison to a vice versa agreement. Additionally, in MZ twins, eating more snacks was associated with a higher BMI, while eating more healthy food, having a regular eating pattern and being concerned about one's appearance were linked with a lower BMI. Similar behaviors have been associated with BMI in MZ (Bogl et al., 2009; Pietiläinen et al., 2010; Rissanen et al., 2002) and DZ (Bogl et al., 2009) twin pairs. In these studies, no link emerged between eating regularly and BMI, except one reported an association of obesity with a higher intake of sweet and fatty delicacies (Bogl et al., 2009). None of these studies, including ours, found clear differences in BMI based on sweet consumption. Evidence on the associations between sugar intake and body weight remains inconsistent (van Baak \& Astrup, 2009).

In the food diaries, the leaner and heavier co-twins of the BMIdiscordant pairs reported similar dietary intakes, approximately in line with the Nordic Nutrition Recommendations (Nordic Council of Ministers, 2014). However, it is likely that the heavier co-twins significantly underreported, as shown with the doubly labeled water method in our previous sample of BMI-discordant MZ twin pairs (Pietiläinen et al., 2010). Furthermore, undereating during dietary recording periods is a common reason for dietary misreporting, especially by those experiencing obesity (Goris et al., 2000).
The current study did not consider energy expenditure, achieved largely through physical activity (PA). In our earlier study, the one on PA and metabolic outcomes, we investigated approximately 25 of the same MZ BMI-discordant twin pairs included here (Berntzen et al., 2018). The heavier co-twins took on average nearly 2000 fewer steps per day, and performed approximately $15 \mathrm{~min}$ less moderate to vigorous PA. Therefore, the PA deficiency in the heavier co-twins likely contributes to the presence of BMI discordance in these twin pairs. This may also partly explain the lower than expected caloric intake of the heavier co-twins.

The current study suggests that a direct question addressing the subjective ability to regulate food intake may be more reliable in screening obesity-related eating patterns in young adults than are food diaries. Additionally, the disinhibited eating measure (TFEQ) might serve as a comprehensive observational tool to capture relevant motives for overeating. Future research should explore the suitability of the food intake regulation question and the disinhibited eating measure for screening and diagnostic purposes, complemented by intervention studies on these behaviors. For example, incorporating a new healthy habit in daily life may diminish habitual disinhibition (Lillis et al., 2016; Rock et al., 2017). Another focus could be on flexible control of eating behavior, as this was found to diminish the effect of habitual disinhibition on BMI (Hays \& Roberts, 2008). Besides this, upcoming studies should try to implement surveys similar to the co-twin comparison questionnaire in populations other than twins; for example, through inclusion of individuals who can serve as reliable proxy informants for the eating behavior of the participants (e.g. spouse, sibling, other relative or close friend).

This study has strengths and limitations. The design was crosssectional, so no inferences can be made on causality between eating behavior and BMI. Information on their socioeconomic status was unavailable and was therefore absent as a potential confounder in the models. In general, however, twin pairs have a high concordance for educational attainment and socioeconomic status (Marks, 2017; Silventoinen et al., 2000). The co-twin control design is unique, but due to the rarity of BMI-discordant pairs the sample size was small (providing low statistical power). Earlier reports on similar eating behaviors in twins who vary in BMI exist, however, with even smaller sample sizes (Pietiläinen et al., 2010; Rissanen et al., 2002). We applied more lenient inclusion criteria to reach a larger sample size. Instead of a difference in an internationally defined cutoff point of BMI (e.g. healthy weight vs. obesity), we considered any minimum of a 3-point difference in BMI important (averaging about $10 \mathrm{~kg}$ difference in a person with a height of 170 $\mathrm{cm}$ ). For example, within the healthy weight category, a BMI of 24 versus a BMI of 20 increases risk for type II diabetes (Lehtovirta et al., 2010). Beyond the slightly increased sample size, our study investigated for the first time in such a twin design (to our knowledge) the DEBQ, the comprehensive version of the TFEQ and the subtypes of behavioral traits from the TFEQ. None of the questionnaires in our study were previously studied in DZ BMI-discordant twin pairs, except the co-twin comparison questionnaire (Bogl et al., 2009). Differences in anthropometry appeared between $\mathrm{MZ}$ and DZ twins, possibly explained by a genetic pressure for similarity in $\mathrm{MZ}$ pairs. Consequently, discordance in weight is more likely to occur at higher age in MZ pairs. Higher age in itself links with weight gain, which may explain the mild overweight in the leaner co-twins of MZ but not DZ pairs. We performed many tests and reported nominal $p$ values of the differences with conservative nonparametric tests (Sullivan \& Artino, 2013). Perhaps, a multiple 
testing correction could have been applied. However, we tested behavioral traits only by BMI discordance, so no exhaustive associations between behaviors and potentially irrelevant outcome measures were performed to force an appearance of low $p$ values. A multiple testing correction would be overly conservative and could promote type II errors in a small cohort.

We included several validated and reliable questionnaires, and were thus able to examine a multitude of eating behavioral aspects within the same research population. This established a robust and comprehensive overview of variations in eating behavioral dimensions associated with BMI discordance, regardless of numerous personal (age, sex, genes etc.) and shared environmental (in utero, childhood, socioeconomic, neighborhood environment) factors.

\section{Conclusions}

Overeating - measured by 'frequent overeating', 'disinhibited eating' and 'binge-eating score' - emerged as the main behavior associated with higher BMI. The twins agreed more frequently that their heavier co-twins habitually ate more food, and particularly more fatty food. Furthermore, the heavier co-twins were generally less satisfied with their bodies. These findings were independent of genetic and shared environmental influences.

Supplementary material. To view supplementary material for this article, please visit https://doi.org/10.1017/thg.2019.43

Acknowledgments. We would like to thank Alyce M. Whipp MSc for the language revision.

Funding. This study was supported by the Academy of Finland, Centre of Excellence in Research on Mitochondria, Metabolism and Disease (FinMIT), grant number: 272376; Academy of Finland, Centre of Excellence in Complex Disease Genetics, grant numbers: 129680 and 213506; Academy of Finland, grant numbers: 314383, 266286, 141054, 263278, 264146, and 265240; Finnish Medical Foundation; Gyllenberg Foundation; Finnish Foundation for Cardiovascular Research; Novo Nordisk Foundation; University of Helsinki; Helsinki University Hospital; Finnish Diabetes Research Foundation; National Institute of Alcohol Abuse and Alcoholism, grant numbers: AA-09203 and AA-12502; Orion Research Foundation; Juho Vainio Foundation; and Yrjö Jahnsson foundation.

\section{Conflict of interest. None.}

Ethical approval. The authors assert that all procedures contributing to this work comply with the ethical standards of the relevant national and institutional committees on human experimentation and with the Helsinki Declaration of 1975 , as revised in 2008 .

\section{References}

Altman, D. G., \& Bland, J. M. (2011). How to obtain the confidence interval from a $\mathrm{P}$ value. $B M J, 343, \mathrm{~d} 2090$.

Berntzen, B., Jukarainen, S., Kataja, M., Hakkarainen, A., Lundbom, J., Lundbom, N., .. P Pietiläinen, K. (2018). Physical activity, cardiorespiratory fitness, and metabolic outcomes in monozygotic twin pairs discordant for body mass index. Scandinavian Journal of Medicine \& Science in Sports, 28, 1048-1055.

Bogl, L. H., Pietiläinen, K. H., Rissanen, A., \& Kaprio, J. (2009). Improving the accuracy of self-reports on diet and physical exercise: The co-twin control method. Twin Research and Human Genetics, 12, 531-540.

Bohrer, B. K., Forbush, K. T., \& Hunt, T. K. (2015). Are common measures of dietary restraint and disinhibited eating reliable and valid in obese persons? Appetite, 87, 344-351.

Bond, M. J., McDowell, A. J., \& Wilkinson, J. Y. (2001). The measurement of dietary restraint, disinhibition and hunger: An examination of the factor structure of the Three Factor Eating Questionnaire (TFEQ). International Journal of Obesity and Related Metabolic Disorders, 25, 900-906.

Bryant, E. J., King, N. A., \& Blundell, J. E. (2007). Disinhibition: Its effects on appetite and weight regulation. Obesity Reviews, 9, 409-419.

Bublitz, M. G., Peracchio, L. A., \& Block, L. G. (2010). Why did I eat that? Perspectives on food decision making and dietary restraint. Journal of Consumer Psychology, 20, 239-258.

Finnish Food Composition Database. (2009). Release 9. Helsinki, Finland: National Institute for Health and Welfare.

French, S. A., Epstein, L. H., Jeffery, R. W., Blundell, J. E., \& Wardle, J. (2012). Eating behavior dimensions. Associations with energy intake and body weight: A review. Appetite, 59, 541-549.

Garner, D. M. (1991). Eating disorder inventory-2: Professional manual. Odessa, FL: Psychological Assessment Resources.

Goris, A. H., Westerterp-Plantenga, M. S., \& Westerterp, K. R. (2000). Undereating and underrecording of habitual food intake in obese men: Selective underreporting of fat intake. The American Journal of Clinical Nutrition, 71, 130-134.

Gormally, J., Black, S., Daston, S., \& Rardin, D. (1982). The assessment of binge eating severity among obese persons. Addictive Behaviors, 7, 47-55.

Grimm, E. R., \& Steinle, N. I. (2011). Genetics of eating behavior: Established and emerging concepts. Nutrition Reviews, 69, 52-60.

Hakala, P., Rissanen, A., Koskenvuo, M., Kaprio, J., \& Rönnemaa, T. (1999). Environmental factors in the development of obesity in identical twins. International Journal of Obesity and Related Metabolic Disorders, 23, 746-753.

Hamilton, A. S., \& Mack, T. M. (2000). Use of twins as mutual proxy respondents in a case-control study of breast cancer: Effect of item nonresponse and misclassification. American Journal of Epidemiology, 152, 1093-1103.

Hays, N. P., \& Roberts, S. B. (2008). Aspects of eating behaviors 'disinhibition' and 'restraint' are related to weight gain and bmi in women. Obesity (Silver Spring), 16, 52-58.

Hyland, M. E., Irvine, S. H., Thacker, C., Dann, P. L., \& Dennis, I. (1989). Psychometric analysis of the Stunkard-Messick Eating Questionnaire (SMEQ) and comparison with the Dutch Eating Behavior Questionnaire (DEBQ). Current Psychology, 8, 228-233.

Johnson, F., Pratt, M., \& Wardle, J. (2012). Dietary restraint and self-regulation in eating behavior. International Journal of Obesity, 36, 665-674.

Jukarainen, S., Heinonen, S., Rämö, J. T., Rinnankoski-Tuikka, R., Rappou, E., Tummers, M., . . . Pietiläinen, K. H. (2016). Obesity is associated with low NAD(+)/SIRT pathway expression in adipose tissue of bmi-discordant monozygotic twins. The Journal of Clinical Endocrinology and Metabolism, $101,275-283$.

Kaprio, J. (2013). The Finnish Twin Cohort study: An update. Twin Research and Human Genetics, 16, 157-162.

Keski-Rahkonen, A., Bulik, C. M., Neale, B. M., Rose, R. J., Rissanen, A., \& Kaprio, J. (2005). Body dissatisfaction and drive for thinness in young adult twins. The International Journal of Eating Disorders, 37, 188-199.

Keski-Rahkonen, A., Bulik, C. M., Pietiläinen, K. H., Rose, R. J., Kaprio, J., \& Rissanen, A. (2007). Eating styles, overweight and obesity in young adult twins. European Journal of Clinical Nutrition, 61, 822-829.

Lauzon, B. de, Romon, M., Deschamps, V., Lafay, L., Borys, J.-M., Karlsson, J., .. Fleurbaix Laventie Ville Sante Study Group. (2004). The Three-Factor Eating Questionnaire-R18 is able to distinguish among different eating patterns in a general population. The Journal of Nutrition, 134, 2372-2380.

Lehtovirta, M., Pietiläinen, K. H., Levälahti, E., Heikkilä, K., Groop, L., Silventoinen, K., ... Kaprio, J. (2010). Evidence that BMI and type 2 diabetes share only a minor fraction of genetic variance: A follow-up study of 23,585 monozygotic and dizygotic twins from the Finnish Twin Cohort Study. Diabetologia, 53, 1314-1321.

Lillis, J., Niemeier, H. M., Thomas, J. G., Unick, J., Ross, K. M., Leahey, T. M., .. Wing, R. R. (2016). A randomized trial of an acceptance based behavioral intervention for weight loss in people with high internal disinhibition. Obesity (Silver Spring), 24, 2509-2514.

Loos, R. J. (2018). The genetics of adiposity. Current Opinion in Genetics \& Development, 50, 86-95.

Lowe, M. R., \& Levine, A. S. (2005). Eating motives and the controversy over dieting: Eating less than needed versus less than wanted. Obesity Research, 13, 797-806. 
Lowe, M. R., Whitlow, J. W., \& Bellwoar, V. (1991). Eating regulation: The role of restraint, dieting, and weight. International Journal of Eating Disorders, 10 , 461-471.

Marks, G. N. (2017). The contribution of genes and the environment to educational and socioeconomic attainments in Australia. Twin Research and Human Genetics, 20, 281-289.

Mashie FoodTech Solutions AB. (2017). AivoDiet ver 2.1.0.1. Malmö, Sweden: Author.

McGraw, K. O., \& Wong, S. P. (1992). A common language effect size statistic. Psychological Bulletin, 111, 361-365.

Nordic Council of Ministers. (2014). Nordic Nutrition Recommendations 2012 : Integrating nutrition and physical activity. Retrieved from http:// urn.kb.se/resolve?urn=urn:nbn:se:norden:org:diva-2561

Ouwens, M. A., van Strien, T., \& van der Staak, C. P. F. (2003). Tendency toward overeating and restraint as predictors of food consumption. Appetite, 40, 291-298.

Pietiläinen, K. H., Korkeila, M., Bogl, L. H., Westerterp, K. R., Yki-Järvinen, H., Kaprio, J., \& Rissanen, A. (2010). Inaccuracies in food and physical activity diaries of obese subjects: Complementary evidence from doubly labeled water and co-twin assessments. International Journal of Obesity, $34,437-445$.

Rissanen, A., Hakala, P., Lissner, L., Mattlar, C.-E., Koskenvuo, M., \& Rönnemaa, T. (2002). Acquired preference especially for dietary fat and obesity: A study of weight-discordant monozygotic twin pairs. International Journal of Obesity and Related Metabolic Disorders, 26, 973-977.

Rock, C. L., Flatt, S. W., Nichols, J. F., Pakiz, B., Barkai, H. S., Wing, D. R., ... Buehler, A. E. (2017). Changes in disinhibition, restraint and hunger and associated characteristics during a weight loss intervention. Journal of Obesity \& Weight Loss Therapy, 7, 348.

Rönnemaa, T., Koskenvuo, M., Marniemi, J., Koivunen, T., Sajantila, A., Rissanen, A., ... Kaprio, J. (1997). Glucose metabolism in identical twins discordant for obesity. The critical role of visceral fat. The Journal of Clinical Endocrinology and Metabolism, 82, 383-387.

Schaumberg, K., Anderson, D. A., Anderson, L. M., Reilly, E. E., \& Gorrell, S. (2016). Dietary restraint: What's the harm? A review of the relationship between dietary restraint, weight trajectory and the development of eating pathology. Clinical Obesity, 6, 89-100.

Silventoinen, K., Kaprio, J., \& Lahelma, E. (2000). Genetic and environmental contributions to the association between body height and educational attainment: A study of adult Finnish twins. Behavior Genetics, 30, 477-485.
Stunkard, A. J., \& Messick, S. (1985). The three-factor eating questionnaire to measure dietary restraint, disinhibition and hunger. Journal of Psychosomatic Research, 29, 71-83.

Sullivan, G. M., \& Artino, A. R. (2013). Analyzing and interpreting data from Likert-type scales. Journal of Graduate Medical Education, 5, 541-542.

Swinburn, B., Sacks, G., \& Ravussin, E. (2009). Increased food energy supply is more than sufficient to explain the US epidemic of obesity. The American Journal of Clinical Nutrition, 90, 1453-1456.

Timmerman, G. M. (1999). Binge eating scale: Further assessment of validity and reliability. Journal of Applied Biobehavioral Research, 4, 1-12.

van Baak, M. A., \& Astrup, A. (2009). Consumption of sugars and body weight. Obesity Reviews, 10 (Suppl. 1), 9-23.

van Strien, T. (2008). Eating less than required versus eating less than desired. The criterion problem in the validity studies of Williamson et al. (2007). Appetite, 50, 548-549.

van Strien, T., Frijters, J. E. R., Bergers, G. P. A., \& Defares, P. B. (1986). The Dutch Eating Behavior Questionnaire (DEBQ) for assessment of restrained, emotional, and external eating behavior. International Journal of Eating Disorders, 5, 295-315.

van Strien, T., Herman, C. P., \& Verheijden, M. W. (2012). Eating style, overeating and weight gain. A prospective 2-year follow-up study in a representative Dutch sample. Appetite, 59, 782-789.

Vartanian, L. R., Wharton, C. M., \& Green, E. B. (2012). Appearance vs. Health motives for exercise and for weight loss. Psychology of Sport and Exercise, 13, 251-256.

Vitaro, F., Brendgen, M., \& Arseneault, L. (2009). The discordant MZ-twin method: One step closer to the holy grail of causality. International Journal of Behavioral Development, 33, 376-382.

Weinberger, N.-A., Kersting, A., Riedel-Heller, S. G., \& Luck-Sikorski, C. (2016). Body dissatisfaction in individuals with obesity compared to normal-weight individuals: A systematic review and meta-analysis. Obesity Facts, 9, 424-441.

Westenhoefer, J. (1991). Dietary restraint and disinhibition: Is restraint a homogeneous construct? Appetite, 16, 45-55.

Westenhoefer, J., Stunkard, A. J., \& Pudel, V. (1999). Validation of the flexible and rigid control dimensions of dietary restraint. International Journal of Eating Disorders, 26, 53-64.

Williamson, D. A., Martin, C. K., York-Crowe, E., Anton, S. D., Redman, L. M., Han, H., \& Ravussin, E. (2007). Measurement of dietary restraint: Validity tests of four questionnaires. Appetite, 48, 183-192. 